\title{
Ultrasound-Guided Cryoablation of Renal Grafts Tumors
}

\section{Introduction}

Tumors in renal grafts are rare with an estimated prevalence of $0.18 \%$ (X. Tillou et al. Transplant Rev. 2015 and FM Drudi et al. J Ultrasound. 2019 [1, 2]). An increased incidence of graft tumors may be observed in the future due to increased donor age and prolonged graft survival. Furthermore, the immunosuppressant state of graft recipients may cause the tumor to behave more aggressively (G. Ploussard et al. BJU Int. 2012: 109: 195-9 [3]). In these complex cases, there is a need for a minimally invasive nephron-sparing treatment option that can keep patients free of dialysis.

Here we present two cases of T1a renal cell carcinoma (RCC) in renal grafts that were successfully treated using ultrasound-guided percutaneous cryoablation: a minimally invasive technique where only the tumor is destroyed, and the graft's function is preserved. Furthermore, when comparing percutaneous cryoablation to laparoscopic cryoablation and partial nephrectomy, the risk of treatment-related complications is decreased (T. Klatte et al. JUrology. 2014: 191: 1209-17 [4]).

\section{Case description}

Both tumors were incidentally discovered with ultrasound and the malignant diagnoses were confirmed using histopathology.
Computed tomography $(\mathrm{CT})$ revealed no signs of regional or remote metastases. Different treatment options were discussed at a multidisciplinary team conference, where a decision was made to perform percutaneous ultrasound-guided cryoablation

\section{( Fig. 1, 2).}

For cryoprobe placement and visualization of the ice ball, a GE Logiq 9 (GE Healthcare, Milwaukee, WI, USA) ultrasound system with a curved 4C transducer (1.5-4.5 $\mathrm{MHz}$ ) was used in the first case. In the second case, a GE Logiq E9 (GE Healthcare, Milwaukee, WI, USA) ultrasound system with a curved C2-9 transducer (2-9 MHz) was used. The first case was treated using the SeedNet ${ }^{\circledR}$ cryoablation system (Galil Medical, Arden Hills, Minneapolis, MN, USA) and the second case using the Visual-ICE ${ }^{\circledR}$ cryoablation system (Galil Medical, Arden Hills, Minneapolis, MN, USA). 17-G IceSphere ${ }^{\circledR}$ and IceRod ${ }^{\circledR}$ cryoprobes were used (Galil Medical, Arden Hills, Minneapolis, MN, USA). The treatment consisted of a double freeze-thaw cycle (10 minutes of active freeze followed by 8 min of passive thaw). The ice ball overlapped the tumor margin by a minimum of $5 \mathrm{~mm}$.

\section{Case 1}

A 42-year-old female with a well-functioning ten-year-old graft and an immunosuppression regimen consisting of Ciclosporin $75 \mathrm{mg} \times$ 2/day and Prednisolone $5 \mathrm{mg} \times 1 /$ day. The graft tumor was a $3 \times 4 \times 4 \mathrm{~cm}$ clear cell carcinoma (Fuhrman grade 2 ) with a RENAL score of 7 . The patient was under general anesthesia and placed in a lateral recumbent position during treatment. Hydrodissection with saline was used the remove the tumor from vital organs. The tumor was treated using four IceRod ${ }^{\circledR}$ cryoprobes and one temperature sensor for monitoring. The patient had no complications and was discharged the following day. Creatinine remained stable at a habitual level around $240 \mu \mathrm{mol} / \mathrm{L}$. Magnetic resonance imaging (MRI) performed one month postoperative revealed slight signs of rim enhancement but no signs of incomplete ablation. The fouryear follow-up MRI examinations revealed no signs of recurrence or metastatic disease. The patient refused further follow-up examinations but remains free of dialysis.

\section{Case 2}

A 70-year-old male with a well-functioning 15-year-old graft and an immunosuppression regimen consisting of Tacrolimus $5 \mathrm{mg} \times$ $2 /$ day and Prednisolone $5 \mathrm{mg} \times 1 /$ day. The graft tumor was a $2 \times 2 \times 2 \mathrm{~cm}$ clear cell carcinoma (Fuhrman grade 2 ) with a RENAL score of 8 . The patient was under general anesthesia and placed in supine position during treatment. Hydrodissection with saline was used the remove the tumor from vital organs. The tumor was treated using four lceSphere ${ }^{\circledR}$ cryoprobes. The patient had no
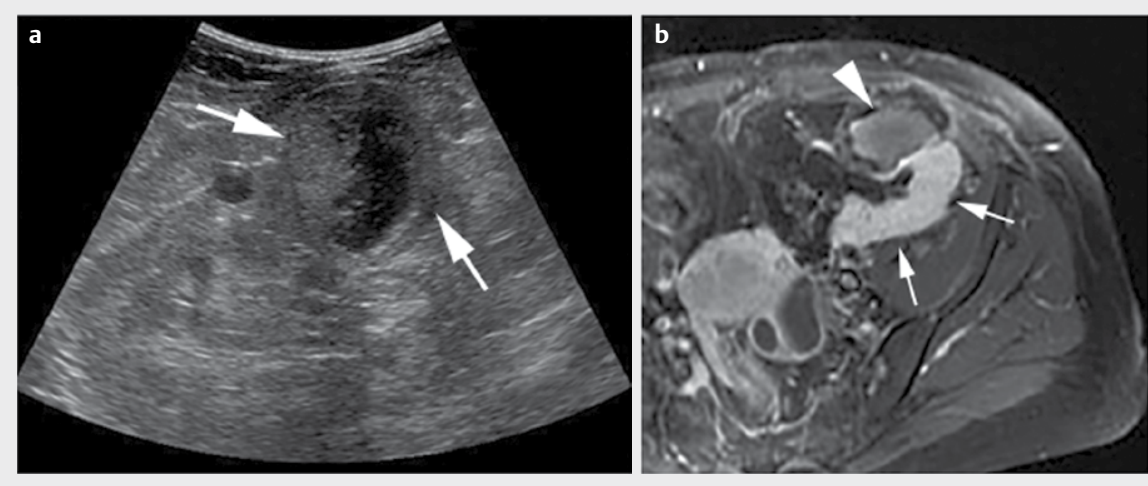

- Fig. 1 Case 1; a Ultrasonography of the tumor in the renal graft (arrow) before CRYO ablation. The graft tumor was a 3x4x4 cm clear cell carcinoma (Fuhrman grade 2). b Six-month follow-up imaging with gadolinium contrast-enhanced T1-weighted MRI demonstrates normal kidney transplant (small arrows) and complete ablation of the tumor (arrowhead). 

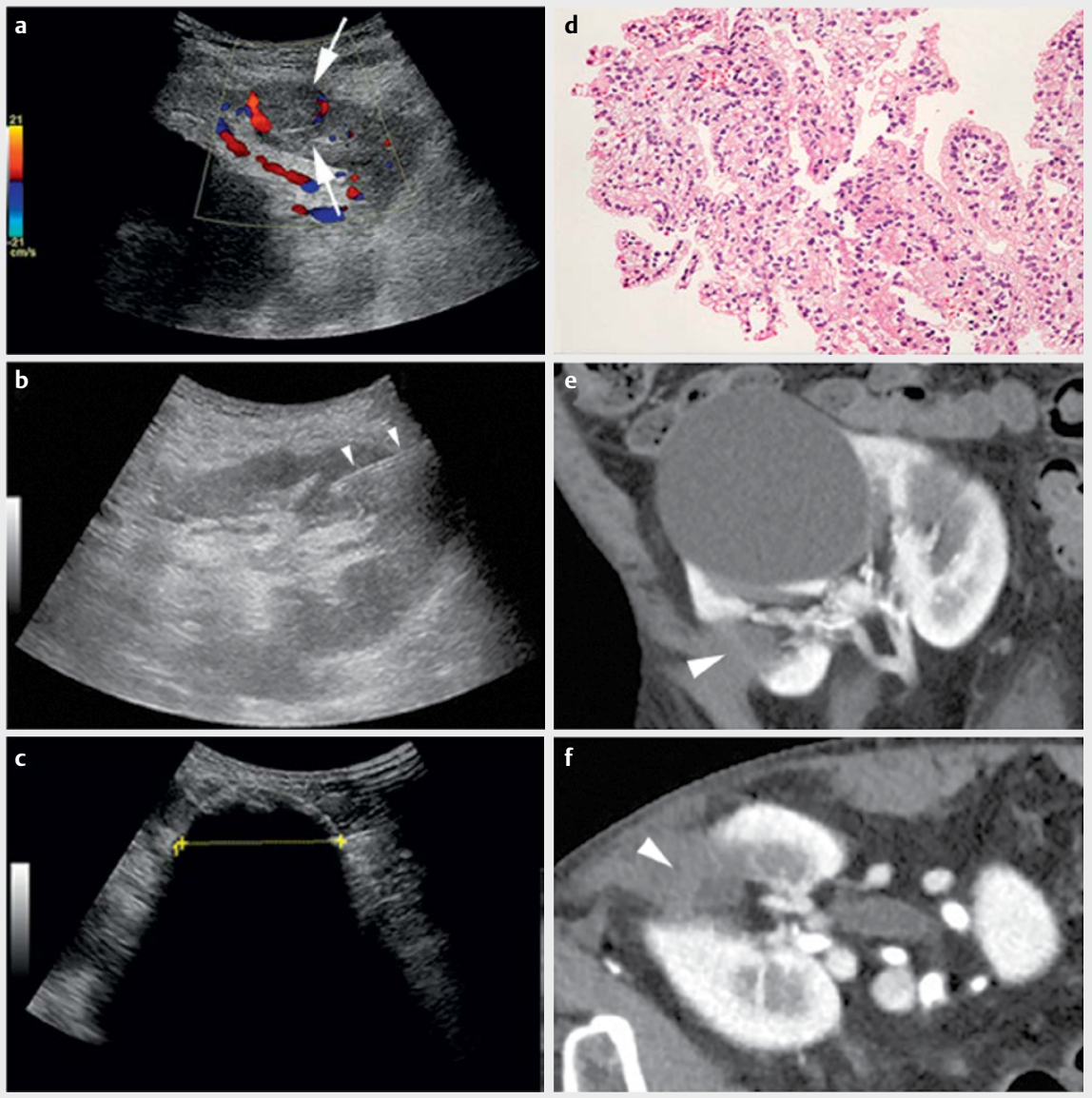

- Fig. 2 Case 2; a Ultrasonography of the tumor in the renal graft (arrow) before CRYO ablation. The graft tumor was a $2 \times 2 \times 2 \mathrm{~cm}$ clear cell carcinoma (Fuhrman grade 2). b Ultrasonography guided placement of the cryoprobe (small arrowhead). $\mathbf{c}$ Ultrasonography of the iceball formation during cryoablation. $\mathbf{d}$ Tumor biopsy shows clear cell carcinoma Fuhrman grade 2 . Six-month follow-up with contrast-enhanced CT in the arterial phase in $\mathbf{e}$ coronal and $\mathbf{f}$ axial sections demonstrate complete ablation of the tumor (arrowhead). Note the large simple cyst in the kidney transplant.

complications and was discharged four hours after treatment. Creatinine level remained stable at a habitual level of $120 \mu \mathrm{mol} / \mathrm{L}$. The one-year follow-up MRI examinations revealed no signs of recurrence or metastatic disease. The patient will be offered annual MRI for a total of five years.

\section{Discussion}

Percutaneous cryoablation could be an effective modality for the treatment of RCC in renal grafts. The sustained renal function and early discharge after treatment seen in the two cases presented in this case report correlate to the findings in a review by Zargar et al. (H. Zargar et al. Eur Urol. 2015 [5]). In this review, the authors conclude that the percutaneous approach in native kidneys has minimal impact on renal function and a low complication rate. However, no randomized studies exist, and the authors conclude that confounding factors and selection bias are an important limitation to their results.

The two cases presented in this case report demonstrate that percutaneous cryoablation is a feasible treatment option for RCC in renal grafts. However, existing literature shows that cryoablation is not commonly used. In a newly published systematic review (E. Favi et al. World J Clin Cases. 2019 [7]) 28 studies were selected, describing a total of 100 ablative therapy (AT) in 92 patients: RFA $(n=78)$, cryoablation $(n=$ 15), MWA ( $n=3), \operatorname{HIFU}(n=3)$, and IRE ( $n=$ $1)$. They concluded that AT for renal allograft neoplasms represents a promising alternative to radical nephrectomy and NSS in carefully selected patients.

Ultrasound visualization of the tumor and surrounding vital organs is mandatory. It is essential that surrounding organs are protected from cryoablation. Posterior and posteromedial RCCs within renal grafts are a challenge to treat with cryoablation. With saline hydrodissection vital organs are often easily removed. Therefore, the majority of all renal graft RCCs are potentially treatable with percutaneous ultrasound-guided cryoablation.

In general, there is a shortage of literature comparing the different guiding methods used for percutaneous cryoablation of renal masses. Ultrasound is accepted as a reliable method of guidance during probe placement. However, ultrasound lacks the ability to monitor ice formation sufficiently, which is possible with CT or MRI (S. Tatli et al. Diagn Interv Radiol. 2010: 16: 90-5 [6]). CT guidance has some disadvantages: radiation exposure for both patient and radiologist, use 
of contrast media, and expense. The latter also applies to MRI.

At our institution ultrasound guidance has been the preferred standard since 2005 , but since 2014 CT guidance has been more frequently used for tumors in native kidneys.

There are few articles discussing the advances of contrast-enhanced ultrasound (CEUS) for the ablative treatment of RCC (PS. Sidhu et al. Ultraschall Med. and V. Cantisani et al. Eur J Radiol. [8, 9]). CEUS will be an indispensable diagnostic tool in the future.

In conclusion, percutaneous ultrasoundguided cryoablation is feasible and should be considered when deciding upon the most appropriate procedure for the treatment of RCC in renal grafts.

\section{Conflict of Interest}

The authors declare that they have no conflict of interest.

\section{Authors}

Janni Lynggård Bo Madsen ${ }^{1}$, Camilla Brinkmann Bak-Ipsen², Tommy Kjærgaard Nielsen ${ }^{3,4}$, Jan Solvig ${ }^{2}$, Ole Graumann ${ }^{1,5}$

\section{Affiliations}

1 Radiology, Odense University Hospital, Odense, Denmark

2 Radiology, Aarhus University Hospital, Aarhus, Denmark

3 Urology, Aarhus University Hospital, Aarhus, Denmark

4 Institute of Clinical Research, Aarhus, Aarhus Universitet, Denmark
5 Department of Clinical Research, Odense, University of Southern Denmark, Denmark

\section{References}

[1] Tillou X, Guleryuz K, Collon S, Doerfler A. Renal cell carcinoma in functional renal graft: Toward ablative treatments. Transplant Rev (Orlando). 2016; 30: 20-66. doi: 10.1016/j.trre.2015.07.001

[2] Drudi FM, Cantisani V, Granata A et al. Multiparametric ultrasound in the evaluation of kidney disease in elderly published online ahead of print, 2019. J Ultrasound. 2019; doi: 10.1007/ s40477-019-00390-5

[3] Ploussard G, Chambade D, Meria P et al. Biopsy-confirmed de novo renal cell carcinoma (RCC) in renal grafts: $A$ single-centre management experience in a 2396 recipient cohort. BJU Int 2012; 109: 195-199

[4] Klatte T, Shariat SF, Remzi M. Systematic review and meta-analysis of perioperative and oncologic outcomes of laparoscopic cryoablation versus laparoscopic partial nephrectomy for the treatment of small renal tumors. J Urol 2014; 191: 1209-1217

[5] Zargar H, Atwell TD, Cadeddu JA et al. Cryoablation for Small Renal Masses: Selection Criteria, Complications, and Functional and Oncologic Results. Eur Urol. 2016; 69: 116-128. doi:10.1016/j.eururo.2015.03.027

[6] Tatli S, Acar M, Tuncali K, Morrison PR, Silverman S. Percutaneous cryoablation techniques and clinical applications. Diagn Interv Radiol 2010; 16: 90-95

[7] Favi E, Raison N, Ambrogi F et al. Systematic review of ablative therapy for the treatment of renal allograft neoplasms. World J Clin Cases 2019; 7 (17): 2487-2504. doi: 10.12998/wjcc.v7.i17.2487
[8] Sidhu PS, Cantisani V, Dietrich CF et al. The EFSUMB Guidelines and Recommendations for the Clinical Practice of Contrast-Enhanced Ultrasound (CEUS) in Non-Hepatic Applications: Update 2017 (Long Version). Die EFSUMB-Leitlinien und Empfehlungen für den klinischen Einsatz des kontrastverstärkten Ultraschalls (CEUS) bei nicht-hepatischen Anwendungen: Update 2017 (Langversion). Ultraschall in Med 2018; 39 (2): e2-e44. doi: 10.1055/a-0586-1107

[9] Cantisani V, Bertolotto M, Weskott HP et al. Growing indications for CEUS: The kidney, testis, lymph nodes, thyroid, prostate, and small bowel. Eur J Radiol 2015; 84 (9): 1675 1684. doi: 10.1016/j.ejrad.2015.05.008

\section{Correspondence}

\section{Dr. Ole Graumann}

Odense Universitetshospital, Radiology, Kløvervænget 10

5000 Odense

Denmark

Tel.: + 4522910914

olegraumann@dadlnet.dk

Bibliography

DOI https://doi.org/10.1055/a-1196-2244

Ultrasound Int Open 2020; 6: E4-E6

(c) Georg Thieme Verlag KG Stuttgart · New York ISSN 2199-7152

\section{(요 (1) $(2)$}

